\title{
Predictive factors of successful salvage microdissection testicular sperm extraction (mTESE) after failed mTESE in patients with non-obstructive azoospermia: Long-term experience at a single institute
}

\author{
Cem Yücel, Salih Budak, Mehmet Zeynel Keskin, Erdem Kisa, Zafer Kozacioglu \\ Department of Urology, Tepecik Training and Research Hospital, Izmir, Turkey.
}

\begin{abstract}
Summary
Objective: To observe the clinical practice of salvage microdissection testicular sperm extraction (mTESE) in patients with non-obstructive azoospermia (NOA) and to determine the factors that may predict the presence of spermatozoa in preoperative salvage mTESE. Methods: We retrospectively reviewed the medical records of 445 patients with the diagnosis of NOA, who had undergone the MTESE operation consecutively in our institution between the dates of March 2008 and June 2017. The study included a total of 49 patients with failure to detect spermatozoa in the first $m T E S E$ and who had then undergone salvage mTESE. In order to investigate the factors that predict the result of salvage $\mathrm{mTESE}$, the patients were classified into two groups according to the outcome of salvage mTESE, as those with and without spermatozoa retrieval. Patients in these two groups were compared with regard to age, body mass index, history of varicocele, history of cryptorchidism, duration of infertility, outcomes of genetic analysis, results of hormone profiles and the testicular histopathology results of the first mTESE. Results: The sperm retrieval rate following salvage mTESE was observed to be $42.8 \%$. Statistically a significant difference was determined between the mean follicle stimulating hormone (FSH) values of the groups ( $p=0.013)$. No significant difference was observed between the groups with regard to the remaining parameters.

Conclusion: It was observed that among the factors that predict the success of sperm retrieval in salvage mTESE in patients with NOA and previous unsuccessful sperm retrieval in mTESE operation, only the pre-operative FSH level was observed to significantly correlate with the success in salvage mTESE.
\end{abstract}

KEY WORDS: Azoospermia; Salvage; Testicular sperm extraction; Spermatozoa.

Submitted 11 January 2018; Accepted 20 February 2108

\section{INTRODUCTION}

Azoospermia is described as the absence of spermatozoa in the ejaculate and is observed in 1\% of all men and 10$15 \%$ of those with the complaint of infertility (1). Azoospermia is examined in two groups according to its etiology as obstructive and non-obstructive azoospermia. Non-obstructive azoospermia (NOA) is accepted as the absence of spermatozoa in the ejaculate due to minimally developed or unproduced cells in the testicles.
The current treatment of NOA is sperm retrieval from the testes via testicular sperm extraction (TESE) and using these sperms in intracytoplasmic sperm injection (ICSI) to obtain a healthy pregnancy (2). Currently, TESE operations are performed in the guidance of a microscope (mTESE). Although it was initially reported that retrieval rate following a first TESE attempt in a well-defined NOA population was around $50 \%$, recovery rates reported subsequently in literature were inconsistent (3).

Unsuccessful sperm retrieval from the first TESE operation results in negative emotional and financial effects. Salvage TESE offers a further chance of pregnancy for whom the first TESE has been unsuccessful. There is no clinical finding or test that precisely predicts the outcome of TESE preoperatively. Knowing the clinical characteristics that help predicting the outcomes of salvage TESE would be facilitative for the preoperative counseling and clinical management of patients undergoing salvage TESE. Currently, there are not many studies that have published the clinical application of salvage TESE in the literature.

The aim of this study was to observe the clinical practice of salvage mTESE in patients with NOA and to determine the factors that may predict the presence of spermatozoa in preoperative salvage mTESE, and help physicians determine the best candidates for this procedure.

\section{Materials AND METHOdS}

Patients and study design

We retrospectively reviewed the medical records of 445 patients with the diagnosis of NOA, who had undergone the MTESE operation in our institution between the dates of March 2008 and June 2017. The diagnosis of NOA was confirmed by clinical findings, medical history, physical examination, serum hormone levels, genetic analysis and as suggested by the WHO guideline, 2 semen analysis. Semen analyses were obtained by masturbation after 3-4 days of sexual abstinence.

The levels of serum total testosterone, follicular stimulating hormone (FSH), luteinizing hormone (LH), estradiol, prolactin of the patients and the genetic analyses 
(karyotype analysis and Y chromosome micro-deletion analysis) results were evaluated. For the hormone profile, blood was drawn from the antecubital vein of the patients after at least 8 hours of fasting. The micro-particle enzyme immuno-assay method (Roche/Hitachi, Cobas e601, Indianapolis, IN, USA) was used to determine all the hormone levels. For the chromosome analysis, the peripheral venous blood samples of the patients were subjected to 72 hours of phytohemagglutinin-induced cell culture.

The study included a total of 49 patients with NOA, who had previously mTESE and no sperm retrieval could be available. All patients included in the study had undergone TESE operations according to the MTESE procedure both in the previous attempt and in the second attempt. In order to ascertain wound healing following the first operation, salvage TESE was planned for a minimum of 3 months afterwards. No hormone therapy was administered to the patients in the time between the first TESE and salvage TESE. Those with a Y chromosome micro-deletion analysis revealing AZFa or AZFb, history of malignancy, those who were morbidly obese, those who had obstruction-related azoospermia, those who had undergone mTESE prior to the second ICSI attempt despite sperm retrieval being possible in the first TESE, and undergone multiple mTESEs, and those whose first TESE operation was conventional, were excluded from the study. The patients were classified into two groups as those with or without sperm retrieval in order to investigate the factors that predict the outcome of salvage mTESE.

Patients in these two groups were compared with regard to age, body mass index (BMI), history of varicocele, history of cryptorchidism, duration of infertility, results of genetic analysis, results of hormone profiles and testicular histopathology results of the first mTESE.

\section{TESE technique}

On the day that the TESE operation was planned, additional sperm samples were obtained and it was confirmed that there were no sperms present. Informed consent was obtained from all of the patients before TESE. All of the patients underwent spinal anesthesia for TESE. A midline scrotal incision was made and the scrotal content was pushed out from the side with the larger testis. The tunica vaginalis was opened and the tunica albuginea that surrounds the testicle was visualized. After this stage, the operation was handled under operating microscope. As described by Schlegel, an avascular area was selected from the antimesenteric area to the tunica albuginea and a $3 \mathrm{~cm}$ incision was made with a thin scalpel (4). Small samples were obtained from opaque, large, white tubules in the testicular parenchyma. Each sample was placed in a Petri dish filled with human tubal fluid. All samples were immediately evaluated by an embryologist using a $200 \mathrm{x}$ magnification microscope in order to investigate the presence of spermatozoa. The operation was terminated when suitable spermatozoa were found for ICSI. If spermatozoa were not detected in the first samples, additional samples were obtained from the same testicle. In cases where the spermatozoa were not found in the samples sent from the larger testis, the sam- ples were also obtained from the contra-lateral testis. The biopsy specimen was sent to the pathology laboratory intraoperatively in order to determine the testicular histopathology.

\section{Histopathological analysis}

In order to define the testicular histopathology, all testicular biopsy samples were fixed within Bouin's solution, and embedded into paraffin blocks following the tissue processing steps. $4 \mu \mathrm{m}$-thick sections were obtained, stained using hematoxylin and eosin dye, and evaluated under a microscope with $400 \mathrm{x}$ magnification by the same pathologist who was experienced in this field for more than 10 years. Germinal epithelia of at least 100 seminiferous tubules were evaluated for each biopsy sample. In the presence of germinal epithelium, the spermatogenetic situation was assessed using the Johnsen's score (JS). According to JS, the tissue maturation and spermatogenetic situation of the germinal epithelia of each sample were scored between 1 and 10. In this scoring system, tubular necrosis was scored as 1, Sertoli cell only was scored as 2, spermatogonia only was scored as 3, arrest at primary spermatocyte was scored as 4 or 5 , arrest at the early spermatid stage was scored as 6 or 7 , arrest at the late spermatid stage was scored as 8 or 9 , and full spermatogenesis was scored as 10 (5). The mean JS was calculated for each sample. Testicular biopsy specimens were classified according to the histopathological criteria as follows: normal spermatogenesis (NS) (mean JS; 10), hypospermatogenesis (HS) (mean JS; 8-9), late maturation arrest (LMA) (mean JS; 6-7), early maturation arrest (EMA) (mean JS; 3-4-5), Sertoli cell only (SCO) (mean JS; 2) and hyalinization of tubules (HT) (mean JS; 1).

\section{Statistical analysis}

The conformity of the variables to the normal distribution was assessed with the Shapiro Wilk test. The categorical variables were described using frequencies with percentages, and the numerical variables were described using the mean and standard deviation values.

The Student's t-test and the chi-square test were used for the intergroup analyses of the continuous variables.

The chi-square test or the Fisher's exact chi-square was used to for the categorical variables. More than two independent averages were compared with the ANOVA test and the Kruskal Wallis test. We performed the univariate and the multivariate analysis to identify the factors associated with and predictive of positive sperm retrieval during a salvage mTESE. Multiple logistic regression analysis was performed using a model including age, FSH and LH levels, and JS. The data analysis was carried out using the Statistical Package for the Social Science (SPSS Inc, Chicago, Illinois, USA) version 22.0 and a $p$ value of $<0.05$ was considered significant.

\section{Results}

In 21 of the 49 participants (42.8\%), sperm retrieval was possible via salvage mTESE. A statistically significant difference was observed between the groups with and without sperm retrieval, with regard to FSH levels $(20.4 \pm 9.7$ vs. $31.2 \pm 10.4$, respectively; $\mathrm{p}=0.013$ ) (Table 1 ). 
Table 1.

Comparison between the successful and unsuccessful sperm retrieval in salvage $\mathrm{MTESE}$.

\begin{tabular}{|lcccc|}
\hline Variables & $\begin{array}{c}\text { Overall } \\
\mathbf{( n = 4 9 )}\end{array}$ & $\begin{array}{c}\text { Spermatozoa } \\
\text { were retrievied } \\
\mathbf{( n = 2 1 )}\end{array}$ & $\begin{array}{c}\text { No Spermatozoa } \\
\text { were retrievied } \\
\text { (n = 28) }\end{array}$ & P value \\
\hline Age (years) & $35.7 \pm 5.1$ & $35.4 \pm 5.9$ & $36.0 \pm 4.9$ & 0.817 \\
\hline Duration of infertility (years) & $6.82 \pm 3.67$ & $6.58 \pm 3.94$ & $7.01 \pm 3.63$ & 0.844 \\
\hline BMl & $22.8 \pm 1.2$ & $23.7 \pm 1.3$ & $22.1 \pm 1.2$ & 0.902 \\
\hline T (ng/dL) & $401.1 \pm 186.5$ & $406.3 \pm 279.0$ & $397.3 \pm 135.8$ & 0.694 \\
\hline E2 (pg/mL) & $31.3 \pm 12.4$ & $30.0 \pm 9.9$ & $32.4 \pm 13.3$ & 0.729 \\
\hline FSH (mIU/mL) & $26.5 \pm 10.2$ & $20.4 \pm 9.7$ & $31.2 \pm 10.4$ & 0.013 \\
\hline LH (mlU/mL) & $11.3 \pm 7.1$ & $8.7 \pm 5.4$ & $13.4 \pm 9.1$ & 0.161 \\
\hline PRL (ng/mL) & $10.3 \pm 3.8$ & $10.5 \pm 4.0$ & $10.2 \pm 3.9$ & 0.834 \\
\hline Mean JS & $5.4 \pm 1.1$ & $4.2 \pm 1.0$ & $6.4 \pm 1.1$ & 0.246 \\
\hline Histopathology (n/\%) & $8(16.3)$ & $2(9.5)$ & $6(21.4)$ & 0.621 \\
HT & $11(22.4)$ & $4(19.0)$ & $7(25)$ & \\
SCO & $11(22.4)$ & $3(14.2)$ & $8(28.5)$ & \\
EMA & $10(20.4)$ & $5(23.8)$ & $5(17.8)$ & $2(7.1)$ \\
LMA & $5(10.2)$ & $3(14.2)$ & $0(0)$ & \\
HS & $4(8.1)$ & $4(19.0)$ & & \\
NS & & & \\
\hline T, testosterone; E2, estradiol; FSH, follicle-stimulating hormone; LH, luteinizing hormone; PRL, prolactin; & \\
HT, hyalinization of tubules; SCO, Sertoli cell only; EMA, early maturation arrest; LMA, late maturation arrest; & \\
HS, hypospermatogenesis; NS, normal spermatogenesis; & & & \\
\hline
\end{tabular}

\section{Table 2.}

Logistic regression analysis model for successful sperm retrieval in salvage $m$ TESE.

\begin{tabular}{|lccc|}
\hline Variables & $\mathbf{O R}$ & $\mathbf{9 5 \%} \mathbf{~ C l}$ & $\mathbf{p}$ value \\
\hline Age & 0.992 & $0.938-1.136$ & 0.657 \\
\hline FSH & 0.963 & $0.944-0.982$ & 0.032 \\
\hline LH & 0.786 & $0.857-1.089$ & 0.356 \\
\hline JS & 0.763 & $0.865-0.979$ & 0.812 \\
\hline $\begin{array}{l}\text { JS, Johnsen's score; FSH, follicle-stimulating hormone; LH, luteinizing hormone; } \\
\text { OR, odds ratio; Cl, confidence interval. }\end{array}$ & \\
\hline
\end{tabular}

No significant difference was observed for the remaining hormone parameters, age, BMI, and duration of infertility. The clinical and laboratory findings of the patients have been presented in Table 1. No history of cryptorchidism was observed in any of the patients included in the study. Non-mosaic Klinefelter's syndrome was observed in a patient prior to salvage mTESE, and no sperm could be obtained in this patient in salvage mTESE. Grade 1-2 varciocele was detected in the physical examination of 4 patients in the sperm retrieval group and 5 patients in the no sperm retrieval group. No serious complication was observed during mTESE or within the post-operative 3 weeks. The mean JS of the 49 participants was found to be 5.4 \pm 1 .1. The JS among the sperm retrieval group and the no sperm retrieval group were $4.2 \pm 1.0$ and $6.4 \pm 1.1$, respectively. The sperm retrieval rates in patients with HT, SCO, MA and HS histopathologies were 25\% (2/8), 36\% (4/11), 38\% (8/21) and 60\% (3/5), respectively. The sperm retrieval rate among patients with NS histopathology in the first mTESE was 100\% (4/4). No statistically significant difference was observed between two groups with regard to testicular histopathology and mean JS ( $\mathrm{p}=0.621 ; \mathrm{p}=0.246$, respectively). The multiple logistic regression analysis was performed by constructing a model including age, FSH and LH levels, and JS. This model has been presented in Table 2. It was observed that FSH was a significant and independent predictive factor for positive sperm retrieval in salvage mTESE ( $p=0.032)$.

\section{Discussion}

In this study, the factors predicting the success of positive sperm retrieval in salvage mTESE in patients with NOA and previous unsuccessful retrieval in MTESE were investigated, and a statistical correlation was observed only between the preoperative FSH level and the success in salvage mTESE. Herein, we have summarized our experience of salvage mTESE in patients with NOA in our single unit within an 9-year interval. There are only five studies investigating the factors predicting the success of salvage mTESE in patients with NOA and previous unsuccessful mTESE in the literature. In these studies, the sperm retrieval rates were reported to be between $30 \%$ and $46 \%$.

Table 3.

Summary of the studies investigating the factors predicting the success of salvage TESE in patients diagnosed with NOA, who had previously undergone unsuccessful TESE.

\begin{tabular}{|c|c|c|c|c|c|c|c|c|c|}
\hline Variables & $\begin{array}{l}\text { Initial TESE } \\
\text { procedure }\end{array}$ & $\begin{array}{l}\text { Salvage TESE } \\
\text { procedure }\end{array}$ & N & $\begin{array}{l}\text { SRR in } \\
\text { the study }\end{array}$ & $\begin{array}{l}\text { SRR in the } \\
\text { HT group }\end{array}$ & $\begin{array}{l}\text { SRR in the } \\
\text { SCO group }\end{array}$ & $\begin{array}{l}\text { SRR in the } \\
\text { MA group }\end{array}$ & $\begin{array}{l}\text { SRR in the } \\
\text { HS group }\end{array}$ & $\begin{array}{l}\text { SRR in the } \\
\text { NS group }\end{array}$ \\
\hline Okuba et al. (2002) & Conventional & Microscopic & 13 & $30.7 \%$ & - & - & - & - & - \\
\hline Tsujimara et al. (2006) & Conventional & Microscopic & 46 & $45.7 \%$ & - & $39.1 \%$ & $41.7 \%$ & $100 \%$ & - \\
\hline Ramasamy and Schlegel (2007) & Conventional & Microscopic & 20 & $45 \%$ & - & $34.3 \%$ & $61.55 \%$ & $93.3 \%$ & - \\
\hline Kalsi et al. (2015) & Conventional & Microscopic & 58 & $46.55 \%$ & - & $40 \%$ & $36.36 \%$ & $75 \%$ & - \\
\hline Xu et al. (2016) & Conventional & Microscopic & 52 & $38.5 \%$ & $25 \%$ & $5.5 \%$ & $25 \%$ & $83.3 \%$ & - \\
\hline This study & Microscopic & Microscopic & 49 & $42.8 \%$ & $25 \%$ & $36 \%$ & $38 \%$ & $60 \%$ & $100 \%$ \\
\hline
\end{tabular}


Among those, the widest study included 58 patients. The outcomes of these studies have been summarized in Table 3 (6-10). In all of these studies, the first unsuccessful TESE was performed via the conventional procedure, whereas salvage TESE was performed with the guidance of a microscope. Therefore, our study is the first to investigate the factors affecting the success of salvage mTESE in patients with NOA and previous unsuccessful TESE performed using the microscopic procedure.

Although empirical medical treatments may be given in patients with NOA prior to MTESE in order to increase the success rates, the efficacies of these treatments have not been confirmed via randomized controlled studies (11). Some authors claim that the possibility to retrieve sperm in salvage TESE may be increased via hormone therapies using clomiphene or human chorionic gonadotropin (HCG) following unsuccessful TESE (12). However, due to the low level of evidence and lack of recommendations for these therapies in the guidelines, no hormone therapy was performed in the time between the first unsuccessful and salvage mTESEs.

There are conflicting data about the effect of FSH level on the success of mTESE in the literature. There are studies demonstrating no effect of FSH on the success of mTESE, whereas there are others demonstrating the contrary (13). FSH acts by binding to its receptors on the Sertoli cells, which are important for spermatogenesis in the testis. Therefore, it tends to decrease in patients with impaired spermatogenesis. Although high FSH levels were related to global impairment of spermatogenesis, there may be normal foci of spermatogenesis in the testicles of these patients (14). Xu et al. and Kalsi et al. have reported no significant difference between the FSH levels of the patients with or without sperm retrieval in salvage $\operatorname{TESE}(9,10)$. On the contrary, in our study, the FSH levels in patients with no sperm retrieval in salvage TESE was observed to be higher.

TESE is an invasive procedure that may lead to complications such as hematoma, infection, fibrosis and even permanent devascularisation. Diagnostic testicular biopsy has complications similar to the mTESE operation. Furthermore, sperm retrieval in subsequent mTESE cannot be assured by retrieved sperm in diagnostic testicular biopsy in patients with NOA. Diagnostic testicular biopsy has not been recommended in clinical practice due to the additional cost, repetitive surgical procedures and the invasive nature of the procedure that increase the risk of complications (15). Thus, the diagnostic testicular biopsy procedure is not being performed in our clinics prior to TESE. Testicular biopsy samples have been obtained during the initial TESE surgery. Additionally, one of the strong aspects of our study was that the testicular histopathology was evaluated by the same and experienced pathologist.Tsujimara et al., Ramasay et al., and Kalsi et al. have evaluated testicular histopathology by classifying into SCO, MA and HS subgroups in order to assess its predictive value for the outcome of salvage TESE (7-9). In addition to these three studies, $\mathrm{Xu}$ et al. have evaluated HT testicular histopathology as an individual subgroup (10).

In contrast to these studies, the NS testicular histopathology subgroup was individually evaluated in our study, and patients with MA were divided into the LMA and EMA groups. This classification was made using the JS in our study and therefore, testicular histopathology is believed to be subgrouped more accurately. Tsujimara et al., Ramasay et al. and Kalsi et al. compared testicular histopathologies, and determined the sperm retrieval rates of $39.1 \%, 34.3 \%$ and $40 \%$, respectively, in patients with SCO histopathology prior to salvage TESE (7-9). In our study, the sperm retrieval rate was 36\% in patients with SCO histopathology. In the study of $X u$ et al., sperm retrieval was possible in $25 \%$ of the patients with HT histopathology in salvage TESE (10). Similiarly, in our study, the sperm retrieval rate among patients with HT histopathology was $25 \%$. These results demonstrate that the possibility of sperm retrieval continues in salvage mTESE even after a previous unsuccessful TESE. In our study, the sperm retrieval rate in salvage mTESE in patients with NS histopathology was 100\% and it was $60 \%$ in patients with HS histopathology. Compared to the other studies in the literature, the sperm retrieval rate among patients in the HS subgroup of our study was lower. The reason for this difference may be the different subgrouping in our study to that in the literature. It was also concluded that the testicular histopathology was not a predictive factor for the success of salvage TESE. The sample size in our study and those in other studies in the literature were small, it is believed that accurate results may be accessed via meta-analyses performed in the future.

Our study has some limitations. First, it was a retrospective study. The effects of cryptorchidism and Klinefelter's syndrome could not be evaluated in salvage TESE, since there was no history of cryptorchidism and only one patient had a history of Klinefelter's syndrome in our study. Although short-term complications following salvage TESE were evaluated, no long-term evaluation was carried out, which is the second limitation of our study. Not all sperms retrieved in TESE can be used in the ICSI procedure. Reproductive analysis of the patients following ICSI was not included in our study, which may be considered as another limitation; however, the outcomes of ICSI are affected by many factors including those of the women as well, and since the priority of our study was the factors affecting the success in salvage MTESE, the ICSI results were not included in the study.

\section{Conclusions}

Our study suggests that salvage mTESE is a safe alternative treatment method in patients with NOA and previous unsuccessful TESE, since the sperm retrieval rate was relatively higher. Evaluation of the preoperative FSH levels may be useful in determining the best candidates for this patient group. Further multi-center, prospective studies with larger sample sizes should be conducted in order to better understand the subject.

\section{ACKNOWLEDGEMENTS}

We would like to thank to Mustafa Karabicak, Ertan Can, Ozgur Cakmak for their contribution to the statistical analysis and Can Kose, Ulku Kucuk, Gokhan Koc for their contribution to the acquisition of data. 


\section{ReFEREnCES}

1. Keskin MZ, Budak S, Aksoy EE, et al. Investigation of the effect of body mass index (BMI) on semen parameters and male reproductive system hormones. Arch Ital Urol Androl. 2017; 89:219-21.

2. Yucel C, Keskin MZ, Cakmak O, et al. Predictive value of preoperative inflammation-based prognostic scores (neutrophil-to-lymphocyte ratio, platelet-to-lymphocyte ratio, and monocyte-toeosinophil ratio) in testicular sperm extraction: a pilot study. Andrology. 2017; 5:1100-4.

3. Vloeberghs $V$, Verheyen $G$, Haentiens $P$, et al. How successful is TESE-ICSI in couples with non-obstructive azoospermia? Hum Reprod. 2015; 30:1790-1796.

4. Schlegel PN. Testicular sperm extraction: microdissection improves sperm yield with minimal tissue excision. Hum Reprod. $1999 ; 14: 131-5$

5. Ustuner M, Yllmaz H, Yavuz U, et al. Varicocele repair improves testicular histology in men with nonobstructive azoospermia. Biomed Res Int. 2015; 709452.

6. Okada H, Dobashi M, Yamazaki T, et al. Conventional versus microdissection testicular sperm extraction for nonobstructive azoospermia. J Urol. 2002; 168:1063-7.

7. Tsujimura A, Miyagawa Y, Takao T, et al. Salvage microdissection testicular sperm extraction after failed conventional testicular sperm extraction in patients with nonobstructive azoospermia. J Urol. 2006; 175:1446-9.

8. Ramasamy R, Schlegel PN. Microdissection testicular sperm extraction: effect of prior biopsy on success of sperm retrieval. J Urol. 2007; 177:1447-9.

9. Kalsi JS, Shah P, Thum Y, et al. Salvage micro-dissection testicular sperm extraction; outcome in men with non-obstructive azoospermia with previous failed sperm retrievals. BJU Int. 2015; 116:460-5.

10. Xu T, Peng L, Lin X, et al. Predictors for successful sperm retrieval of salvage microdissection testicular sperm extraction (TESE) following failed TESE in nonobstructive azoospermia patients. Andrologia. 2017; 49: e12642.

11. Patel DP, Chandrapal JC, Hotaling JM. Hormone-based treatments in subfertile males. Curr Urol Rep. 2016; 17:1-8.

12. Hussein A, Ozgok Y, Ross L, et al. Optimization of spermatogenesis-regulating hormones in patients with non-obstructive azoospermia and its impact on sperm retrieval: a multicentre study. BJU Int. 2013; 111:110-4.

13. Ramasamy R, Lin K, Gosden LV, et al. High serum FSH levels in men with nonobstructive azoospermia does not affect success of microdissection testicular sperm extraction. Fertil Steril. 2009; 92:590-3.

14. Hung AJ, King P, Schlegel PN. Uniform testicular maturation arrest: a unique subset of men with nonobstructive azoospermia. J Urol. 2007; 178: 608-12.

15. Haimov-Kochman R, Lossos F, Prus D, et al. The value of repeat testicular sperm retrieval in azoospermic men. Fertil Steril. 2009; 91:1401-3.

\section{Correspondence}

Cem Yücel, MD (Corresponding Author)

meclecuy@hotmail.com

Salih Budak, MD

salihbudak1977@gmail.com

Mehmet Zeynel Keskin, MD

zeynel_akd@hotmail.com

Erdem Kisa, MD

drerdemkisa@hotmail.com

Zafer Kozacioglu, MD Associate Prof.

zaferkozacioglu@gmail.com

Tepecik Training and Research Hospital

Yenisehir Mah, Gaziler Cad. No:468, Konak/Izmir, Turkey 